\title{
A comparison of patients with relapsed and chemo-refractory gestational trophoblastic neoplasia
}

\author{
T Powles*,', PM Savage', J Stebbing', D Short', A Young', M Bower', C Pappin', P Schmid' and MJ Seckl' \\ 'Department of Medical Oncology, Charing Cross Gestational Trophoblastic Disease Centre, Hammersmith Hospitals Campus of Imperial College \\ London, Fulham Palace Road, London, W6 8RF, UK
}

\begin{abstract}
The majority of women requiring chemotherapy for gestational trophoblastic disease (GTN) are cured with their initial chemotherapy treatment. However, a small percentage either become refractory to treatment, or relapse after the completion of treatment. This study investigates the characteristics and outcome of these patients. Patients were identified from the Charing Cross Hospital GTD database. The outcome of these patients with relapsed disease was compared to those with refractory disease. Between 1980 and 2004, 1708 patients were treated with chemotherapy for GTN. Sixty (3.5\%) patents relapsed following completion of initial therapy. The overall 5-year survival for patients with relapsed GTN was 93\% (95\% Cl 86- 100\%). The overall survival for patients with low-risk and high-risk disease at presentation, who subsequently relapsed was $100 \%(n=35)$, and $84 \%$ $(n=25)$ (95\% Cl: 66-96\%: $P<0.05)$, respectively. Eleven patients were identified who failed to enter remission and had refractory disease. These patients had a worse outcome compared to patients with relapsed disease (5-year survival 43\% (95\% Cl: I2-73\% $P<0.0$ I)). The outcome of patients with relapsed GTN is good. However, patients with primary chemo-refractory disease do poorly and novel therapies are required for this group of patients.

British Journal of Cancer (2007) 96, 732-737. doi: 10.1038/sj.bjc.6603608 www.bjcancer.com
\end{abstract}

Published online 13 February 2007

(C) 2007 Cancer Research UK

Keywords: gestational trophoblastic neoplasia; relapse; chemotherapy

Gestational trophoblastic disease comprises a spectrum of disorders ranging from the premalignant complete and partial hydatidiform moles to the malignant invasive mole, choriocarcinoma and rare placental site trophoblastic tumours (PSTT). The latter three conditions are also collectively known as gestational trophoblastic tumours or neoplasia (GTN). The majority of patients with GTN are cured with chemotherapy. The intensity of the initial chemotherapy for invasive mole and choriocarcinoma is dependant on the risk factors at presentation, with patients stratified into low- and high-risk prognostic groups (Ngan, 2004). These two groups of patients are treated differently. The low-risk patients receive single-agent therapy, most commonly with methotrexate, whereas high-risk patients are treated with combination chemotherapy, using weekly combination chemotherapy, comprising of etoposide, methotrexate, actinomycin alternating with cyclophosphamide and vincristine (EMA-CO) at our institution (Bagshawe, 1976). The outcome of both the groups of patients is good with 5 -year survival of $100 \%$ for low-risk and $86 \%$ for high-risk patients (Farhat et al, 1996; Bower et al, 1997). However, a small minority of patients with GTN will relapse after the completion of treatment.

Owing to the rarity of the disease and the small number of patients relapsing after treatment, there are few publications

\footnotetext{
*Correspondence: Dr T Powles;

E-mail:Thomas.Powes@bartsandthelondon.nhs.uk

Received 23 November 2006; revised 4 January 2007; accepted 7 January 2007; published online 13 February 2007
}

examining the management and outcome of this group. Additionally, previous studies have combined relapsed patients with those that have progressive disease on chemotherapy (refractory disease) (Lurain and Nejad, 2005; Matsui et al, 2005). However, in other curable cancers such as lymphoma and germ cell tumours, there is evidence that the outcome for relapsed as opposed to primary refractory disease is different (Proctor et al, 2001; McNeish et al, 2004). Here, we separately describe and compare the characteristics, management and outcome of patients who relapse following chemotherapy with those who have primary chemotherapy refractory disease.

\section{PATIENTS AND METHODS}

The Charing Cross GTD database includes full clinical details of women diagnosed with GTN. It was screened to identify women who had primary refractory disease or relapsed after completion of chemotherapy for GTN between 1980 and 2004.

\section{Patients with relapsed disease}

Relapse was defined as two elevated and increasing serum human chorionic gonadotropin (hCG) levels, in the absence of a normal pregnancy, after achieving complete serological remission with chemotherapy. Complete remission was defined as a minimum of 6 weeks of normal hCG values on chemotherapy. At this point, chemotherapy was stopped and patients entered the posttreatment screening programme. The post-treatment screening 
programme involves once weekly blood tests for 6 weeks, then two weekly blood and urine samples up to 6 months, followed by urine samples two weekly for the rest of the first year. From there, urine hCG is measured at increasing intervals up to 5 years. At this point urine is requested every 6 months for life.

\section{Patients with refractory disease to EMA/CO therapy}

This group includes high-risk GTN patients whose hCG had increased, or failed to decrease (plateau) on EMA/CO chemotherapy. Human chorionic gonadotropin levels were assessed twice weekly on treatment according to the protocol. As hCG can initially rise and/or take 2-4 weeks before falling following commencement of chemotherapy, patients were given at least 3 weeks of EMA/CO before a diagnosis of refractory disease could be made.

Refractory disease was defined as:

1. Two or more abnormal and increasing plasma hCG levels, despite receiving standard EMA/CO chemotherapy for at least 3 weeks.

2. Three or more consecutive hCG values that had failed to fall more than $5 \%$ below the preceding hCG level. These patients were considered to have a plateau in their hCG.

Patients with refractory disease went on to receive platinumbased chemotherapy (see later for treatment details). Nine patients with high-risk disease were excluded from this study because they died early from GTN, before a fall in the hCG could be attributed to chemotherapy resistance.

\section{Other exclusion criteria}

Patients with PSTT were excluded from this analysis because the treatment and outcomes for these patients are different (Papadopoulos et al, 2002)

\section{Details at initial diagnosis of GTN}

At initial diagnosis, patients were staged according to the national and internationally accepted criteria for GTN, and then stratified into low- or high-risk groups and treated accordingly (Bagshawe, 1976; Kohorn, 2001). Patients were treated in a uniform manner using the standard well-established treatment protocols of methotrexate/folinic acid and EMA-CO. Women with in the lowrisk group received intramuscular methotrexate with folinic acid. In the event of resistance to methotrexate, characterised by a rise or plateau in the serum hCG, treatment was switched to actinomycin D or the EMA-CO high-risk treatment regimen, depending on the hCG level as described previously (McNeish et al, 2002). The majority of patients with high-risk disease were commenced EMA/CO. However, in the presence of brain or liver metastasis different regimens were used (EMA/CNS (high doses of methotrexate) or EP/EMA (EP = etoposide and cisplatin), respectively) (Rustin et al, 1989; Bower et al, 1997; Newlands et al, 2000).

\section{Investigations and treatment at diagnosis of relapse of GTN}

Patients who relapsed underwent restaging, which included a pelvic Doppler US, CT body and CT or MRI brain. The treatment for patients who relapsed after methotrexate or actinomycin for (low-risk disease) was either surgery alone (if the relapse was isolated) or EMA/CO with or without surgery. For those relapsing after EMA/CO (high-risk disease) treatment was either surgery and/or EP/EMA. Relapsed patients were stratified into relapse after initial high- and low-risk treatment for comparative purposes.

\section{Investigations and treatment at diagnosis of refractory} disease

The details and characteristics of these patients were noted at the time of development of refractory disease. Patients went on to receive platinum-based chemotherapy usually EP/EMA (Newlands et al, 2000).

\section{Statistical methods}

Survival was calculated from the day of diagnosis until death or the date of last follow-up. Overall survival duration curves were plotted according to the method of Kaplan and Meier. Comparison of variables between groups was by $\chi^{2}$ test for nominal variables, and Mann-Whitney $U$-test for non-parametric variables, log-rank tests were used to compare overall survival over time and all $P$-values presented are two-sided. Univariate analysis was performed on the cohort with relapsed disease.

\section{RESULTS}

\section{Patients characteristics (Table 1 and Figure 1)}

From a database of 1708-treated GTN patients, 60 (3.5\%) relapsed after chemotherapy, 35 of 1397 (2\%) low-risk patients relapsed compared to 25 of 311 (8\%) high-risk patients. Eleven patients fulfilled the criteria for disease refractory to EMA/CO chemotherapy.

\section{Details of relapsed patients at initial presentation of GTN (Table 1)}

The characteristics of the 60 patients who relapsed are given in Table 1. At initial presentation, 35 (58\%) patients were in the lowrisk prognostic group and received single-agent methotrexate, eight of these patients became resistant to methotrexate and changed to more intensive chemotherapy two with EMA/CO and six with actinomycin (McNeish et al, 2000).

The remaining 25 (42\%) patients initially presented with highrisk disease and were treated accordingly: 17 received EMA/CO chemotherapy, six patients received platinum containing regimens or other regimens including cisplatin etoposide $(n=1)$, cisplatin etoposide and vincristine $(n=1)$. These other regimens were used because of the presence of extra-pulmonary metastasis, intolerance of methotrexate or treatment predating the routine introduction of EMA/CO chemotherapy.

\section{Details of patients at relapse (Table 2)}

The characteristics of the patients at the time of relapse are shown in Table 2 . The majority of relapses $(73 \%)$ occurred within 1 year of completing initial chemotherapy and only one patient relapsed after more than 5 years of follow-up (Table 3). Human chorionic gonadotropin screening of either blood or urine samples identified $47(78 \%)$ of these relapses. Thirteen represented with symptoms (predominantly amenorrhoea), including two patients who did not comply with screening and presented with symptomatic relapse.

\section{Treatment at relapse of GTN (Table 4)}

Treatment at the time of relapse depended on a number of factors. The most important of which were previous treatment at initial presentation (high- $v s$ low-risk prognostic group) and site of disease.

Most women initially treated with methotrexate for low-risk disease received EMA/CO at relapse, although other regimens were used in five patients due to intolerance of EMA/CO (Table 4). Relapse after initial high-risk chemotherapy was treated with 
Table I Characteristics at initial presentation of disease for patients who developed relapsed GTN $(n=60)$

\begin{tabular}{|c|c|c|c|}
\hline Characteristic & $\begin{array}{l}\text { All relapsed patients (high- and } \\
\text { low- risk at initial diagnosis) }\end{array}$ & $\begin{array}{l}\text { Patients with high-risk disease at } \\
\text { initial diagnosis who relapse }\end{array}$ & Refractory disease \\
\hline Number & 60 & 25 & | | \\
\hline Median prognostic score ( $\mathrm{WHO})$ at diagnosis & 7 (range $2-23$ ) & 9 (range $8-23$ ) & 16 (range 8-26) \\
\hline Median prognostic score (FIGO) & 4 (range I-12) & 7 (range $5-12$ ) & 10 (range 6-18) \\
\hline \multirow[t]{3}{*}{ Antecedent pregnancy } & Term 19 & Term 17 & Term 7 \\
\hline & Abortion 7 & Abortion 5 & Abortion I \\
\hline & Mole 34 & Mole 3 & Mole 3 \\
\hline Median hCG before initial treatment & 21000 (range $31-8 \mid 4000)$ & 66000 (range $350-814000)$ & 380000 (range $79000-1100000$ \\
\hline $\begin{array}{l}\text { Duration between causal pregnancy and initial } \\
\text { treatment }\end{array}$ & 0.4 years (range $0.1-7.3$ years) & 1.1 years (range $0.1-7.3$ years) & 3.2 years (range $0.1-15$ ) \\
\hline \multirow[t]{3}{*}{ Uterine size } & $0-3 \mathrm{~cm}-15$ & $0-3 \mathrm{~cm}-7$ & $0-3 \mathrm{~cm} 5$ \\
\hline & $3-5 \mathrm{~cm}-39$ & $3-5 \mathrm{~cm}-16$ & $3-5 \mathrm{~cm}-4$ \\
\hline & $>5 \mathrm{~cm}-6$ & $>5 \mathrm{~cm}-2$ & $>5 \mathrm{~cm}-2$ \\
\hline \multirow[t]{3}{*}{ Site of metastasis } & None 40 & None 12 & None 0 \\
\hline & Lung only 16 & Lung only 9 & Lung only 7 \\
\hline & Other sites ${ }^{\mathrm{a}} 4$ & Other sites ${ }^{\mathrm{a}} 4$ & Other sites ${ }^{b} 4$ \\
\hline \multirow[t]{3}{*}{ Initial treatment regimens } & Methotrexate 35 & EMA/CO 17 & EMA/CO II \\
\hline & EMA/CO 18 & Platinum-based 7 & \\
\hline & Platinum-based 7 & & \\
\hline
\end{tabular}

GTN = gestational trophoblastic neoplasia; $\mathrm{WHO}=$ World Health Organization. ${ }^{a}$ With or without lung metastasis (includes liver (2), brain ( 1 ) and liver and brain $(\mathrm{I})$. ${ }^{\mathrm{b}}$ Liver, 2 ; brain, 2 .

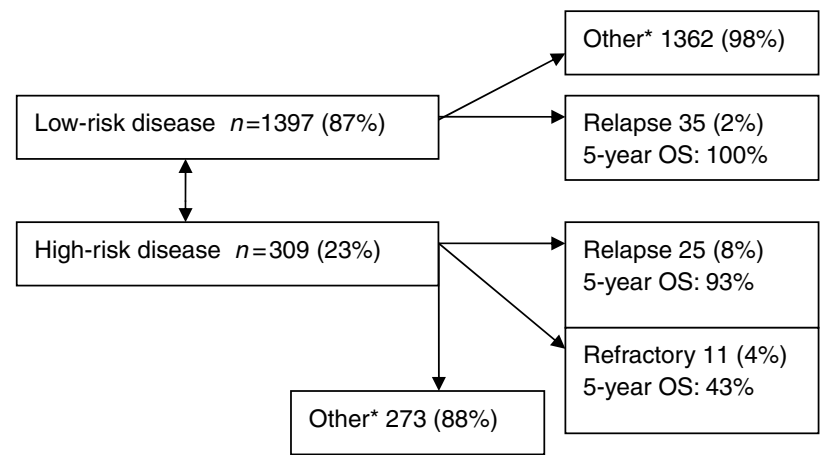

Figure I Origin of patients with relapsed or refractory GTN.

platinum-based chemotherapy in the majority of patients, most frequently the EP/EMA regimen (15 out of 25). EMA/CO was reused successfully at relapse in three patients with a long treatment-free interval ( $>1$ year). Patients with brain metastasis received EMA/CO with high-dose methotrexate (Rustin et al, 1989).

Surgery was performed, in conjunction with chemotherapy, in 15 women with easily resectable metastasis isolated from one organ. Two patients with low-risk disease and isolated uterine relapse underwent a hysterectomy without chemotherapy owing to patient preference, one of these patients relapsed and required chemotherapy and was cured.

There were no treatment-related deaths in this group of patients with relapsed GTN.

\section{Outcome of patients with relapsed GTN (Table 4)}

After a median follow-up of 10.8 years (range 1.7-24.5) after relapse, there have been five deaths among the 60 patients with relapsed disease, all owing to progressive chemotherapy-resistant GTN. These patients received third-line therapy (high-dose treatment in four (McNeish et al, 2004)). Although markers temporarily normalised in two of these patients, none went into a sustained complete remission. Ultimately, all patients progressed and died of progression of disease in multiple sites. All five deaths occurred in patients falling into the initial high-risk prognostic group; none of these patients who died achieved a complete remission with second-line platinum-based chemotherapy. All of these five patients progressed on third-line therapy. Indeed all of the women who went into complete remission after second-line treatment are alive, whereas all patients who progressed on second-line treatment have died of GTN. The 5-year overall survival was $93 \%$ (95\% CI: $68-100 \%)$, this dropped to $84 \%$ (95\%CI: 68-99\%) for patient with high-risk disease. None of the patients initially within the low-risk prognostic group died.

Overall nine patients have relapsed for a second time; three from the initial low-risk disease group and six from the initial high-risk group. The median time interval between first and second relapse was 0.8 years (range $0.3-4.6$ ). All of these patients went into complete remission with third-line treatment and are alive with a median follow-up of 13.0 years (range $3.0-22.3$ ). This third-line treatment included surgery $(n=1)$, chemotherapy $(n=5)$ and chemotherapy and surgery $(n=3)$. The chemotherapy regimens used included high-dose therapy $(n=2)$ and, EP/EMA $(n=5)$, EMA/CO $(n=1)$. Indeed two of these patients have relapsed for a third time and are in remission with fourth-line chemotherapy, with 4.5 and 7.3 years of follow-up.

\section{Prognostic factors for patients with relapsed disease (Table 5)}

A number of clinical variables were found to be of prognostic importance using univariate analysis. These included the presence of metastasis at relapse, where the 5-year overall survival was $79 \%$ (95\%CI: 61 -97) for patients with metastasis and 97\% (95\%CI: 87 100) for patients without metastasis. Other significant factors included a slow hCG-doubling time at relapse, high-risk disease at presentation and term pregnancies. Multivariate analysis was precluded due to the small sample size in conjunction with the small number of events.

\section{Patients with refractory disease (Table 1 and Figure 2)}

Eleven patients progressed on EMA/CO chemotherapy and fulfilled the criteria for refractory disease. Seven of these patients had consecutive rising hCG levels on chemotherapy, whereas the other 
Table 2 Patients characteristics at time of relapse or refractory disease

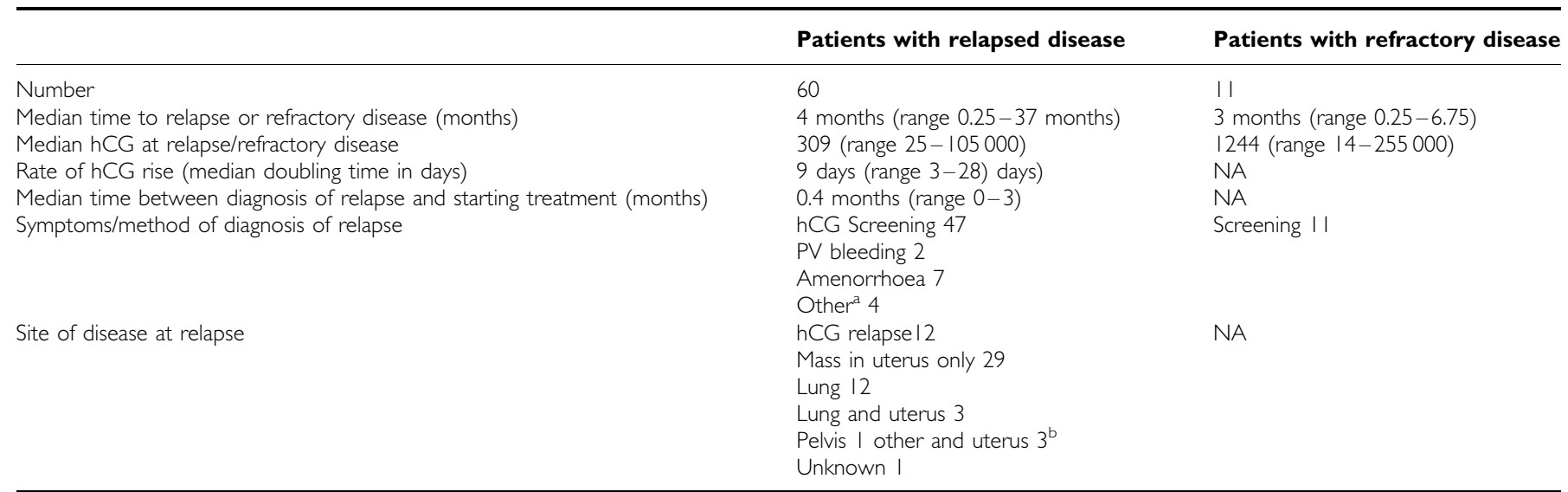

$\mathrm{HCG}=$ human chorionic gonadotropin; NA = not available. a Respiratory symptoms, haemoptysis and re-entry to screening programme. ${ }^{\mathrm{b}}$ Includes liver $(\mathrm{I})$, liver and brain $(\mathrm{I})$, pelvis (2).

Table 3 Time to relapse after chemotherapy

\begin{tabular}{lcc}
\hline Time to relapse & Number (\%) & Cumulative (\%) \\
\hline $0-3$ months & $31(51.6)$ & 51.6 \\
$3-6$ months & $10(16.6)$ & 68.2 \\
$6-12$ months & $3(5)$ & 73.2 \\
$12-24$ months & $7(11.7)$ & 84.9 \\
$1-5$ years & $8(13.5)$ & 98.4 \\
$>5$ years & $1(1.6)$ & 100 \\
\hline
\end{tabular}

four had a plateau in their hCG. All of these patients received platinum-based chemotherapy (EP/EMA 8, EP 3). Six of these patients have died with a median overall survival of 1.2 years. All six patients died of chemoresistant GTN, four of these patients subsequently received high-dose therapy. These patients have a poor prognosis compared to those with relapsed disease after high-risk treatment (5-year survival 43\% (95\% CI: 68-99\%) vs 84\% (95\%CI: $12-73 \%(P=0.003))$. It is noteworthy that these patients had poor prognostic at diagnosis features compared to the high-risk relapse patients (Table 1).

\section{DISCUSSION}

This is the largest reported series of GTN patients with either relapsed disease after chemotherapy or refractory disease. The main findings are that the relapse rate following chemotherapy is low (3.5\%) and the majority of these patients can be cured with further treatment $(>90 \%)$. However, patients with disease which is refractory to high-risk treatment have a significantly less good outcome.

None of the women who relapsed after low-risk disease died, which is reassuring for these patients. Additionally, extrapolating from previously published data, the 5-year survival of patients with relapse after high-risk disease is similar to that for high-risk patients at initial presentation (84\% (95\% CI: 68-99\%) vs $86 \%$ (95\%CI: 82-91\%), respectively) (Bower et al, 1997; Dobson et al, 2000). All of the relapsed patients who died failed to go into complete remission with second-line chemotherapy at relapse. Therefore, obtaining a complete serological remission with second-line chemotherapy is crucial for these patients.

This work also shows the outlook for patients with chemotherapy refractory disease is less good. These findings are consistent
Table 4 Management and outcome of relapsed disease

\begin{tabular}{ll}
\hline Low risk at initial presentation patients $n=35$ & 2 \\
Hysterectomy only & EMA/CO 3 \\
Hysterectomy and chemotherapy & EP I \\
& EMA/CO 25 \\
Chemotherapy only & CO/EP I \\
& EP I \\
& EP/EMA 2 \\
& I00\% \\
5 -year survival & \\
High risk at initial presentation patients $n=25$ & EP/EMA 2 \\
Thoracotomy and chemotherapy & POMB ACE I \\
& EMA/CO I \\
& EP/EMA 3 \\
Hysterectomy and chemotherapy & EMA/CO I \\
& Cis/taxol I \\
& EMA/CNS I \\
Chemotherapy only & EMA/CO 3 \\
& EP/EMA I0 \\
& Cisplatin vincristine I \\
& Cis/taxol I \\
& $84 \%$ (95\% Cl: 68-99\%) \\
\hline
\end{tabular}

EMA/CO - weekly combination chemotherapy, comprising of etoposide(dose), methotrexate, actinomycin alternating with cyclophosphamide and vincristine (Bower et al, 1997). POMBACE - cisplatin, vincristine, methotrexate, bleomycin alternating with actinomycin D, cyclophosphamide and etoposide (Newlands et al, 1983). EP/EMA - etoposide, cisplatin/etoposide, methotrexate and actinomycin D (Newlands et al, 2000). EMA/CNS - etoposide, methotrexate and actinomycin alternating weekly with vincristine and cyclophosphamide. The dose of methotrexate was increased to $\mathrm{Ig} \mathrm{m}^{-2}$. Cis/taxol - cisplatin etoposide and paclitaxel (Osborne et al, 2004) EP cisplatin etoposide (IV day I -5)

with data from other tumour types such as ovary and testis, which demonstrate a difference in outcome regarding patients with refractory and relapsed disease (Thigpen et al, 1993; McCaffrey et al, 1997; Herzog, 2002).

Owing to the lack of published data, there is no specific scoring/ prognostic system for relapsed or refractory patients. The WHO and FIGO scoring systems are used to separate women into high- and low-risk disease at initial presentation. Although these scoring systems do include prognostic scores (points) for previous treatment (up to six points), the scoring system has not been widely evaluated or validated for these rare patients. Although numbers are small, the data presented here do highlight a number 
Table 5 Univariate analysis for patients with relapsed disease

\begin{tabular}{|c|c|c|c|c|}
\hline & & \multicolumn{2}{|c|}{ GTN-related deaths } & $P$-value \\
\hline Median age at relapse (years) & & 32 & 29 & 0.26 \\
\hline \multicolumn{2}{|l|}{ Median hCG at presentation } & 15000 & 28000 & 0.14 \\
\hline \multicolumn{2}{|l|}{ Median interval between causal pregnancy and first chemotherapy } & I.I years & 0.5 years & 0.57 \\
\hline \multicolumn{2}{|l|}{ Maximum hCG at relapse (medium) } & 350 & 121 & 0.41 \\
\hline \multicolumn{2}{|l|}{ HCG doubling time at relapse (days) } & 15 & 7 & $0.01 *$ \\
\hline \multirow[t]{2}{*}{ Nonpulmonary metastasis at presentation } & No & 3 & 52 & $0.05 *$ \\
\hline & Yes & 2 & 3 & \\
\hline \multirow[t]{2}{*}{ Treatment at initial presentation } & High risk & 5 & 20 & $0.01 *$ \\
\hline & Low risk & 0 & 35 & \\
\hline \multirow[t]{2}{*}{ Interval between chemotherapy and relapse } & $0-3$ months & 4 & 30 & 0.19 \\
\hline & $>3$ months & 1 & 25 & \\
\hline Metastasis identified at relapse & No & 1 & 39 & $0.03 *$ \\
\hline \multirow[t]{2}{*}{ Causal pregnancy } & Mole & 0 & 29 & $0.02 *$ \\
\hline & Term & 5 & 26 & \\
\hline
\end{tabular}

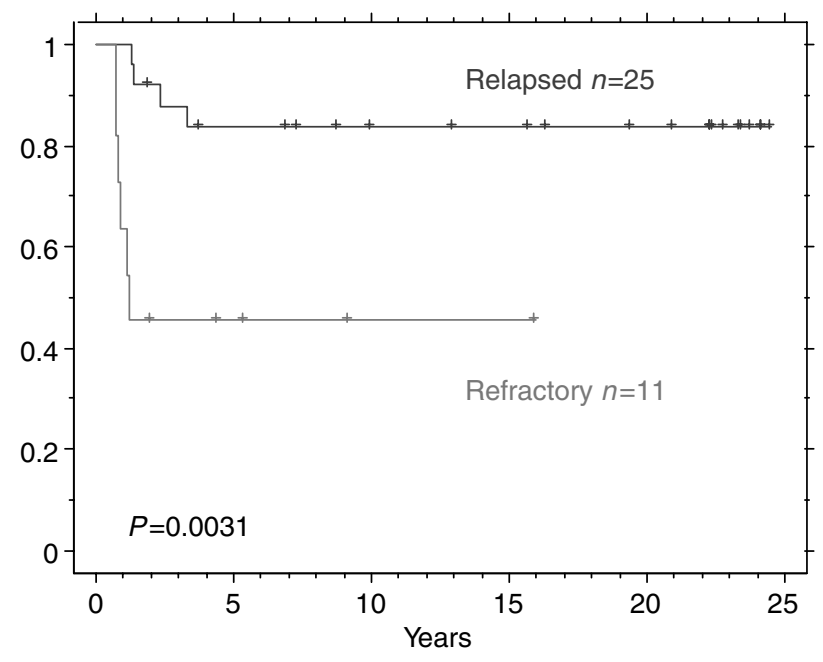

5-year OS

Relapsed $=84 \%$ (95\% Cl: 68-99)

Refractory $=43 \%(95 \% \mathrm{Cl}: 12-73)$

Figure 2 Outcome of patients who relapsed after high-risk treatment vs refractory (progression on initial high-risk chemotherapy).

of factors which were of poor prognostic importance in univariate analysis, these include metastatic disease at relapse, previous highrisk treatment, previous nonpulmonary metastases, nonmolar pregnancies and a slow hCG-doubling time ( $>10$ days). These factors may give us a better insight into predicting the outcome of these patients. For example, none of the women with two or less of these factors have died. Unfortunately, in view of the rarity of the disease and small number of deaths in this cohort, it is not possible to construct and validate a prognostic scoring system.

The observation that patients with a slow hCG-doubling time at relapse appear to have a worse outcome compared to those with a rapid doubling time is interesting. The reasons for this are unclear, but a slow doubling time may be a mark of a slow growing, chemotherapy-resistant tumour or a tumour that no longer secretes hCG appropriately due to mutation or differentiation as has been described with other cancers (Fossa et al, 1992). However, the time between initial diagnosis and relapse was not significant in univariate analysis. This may in part be due to the small numbers because of the rarity of the disease.

Currently, clinicians at the Charing Cross Hospital are treating patients with relapse after methotrexate for low-risk disease with EMA/CO, which seems appropriate as the overall survival is $100 \%$ in this group. EMA/CO is first-line high-risk treatment at the Charing Cross; however, other regimens such as MEA (methotrexate, etoposide and actinomycin) (Dobson et al, 2000) are used at other centres. These appear to have similar relapse and overall survival rates, although direct comparison as part of a study has not been performed. The majority of patients who relapsed after initial high-risk treatment in this series received EP/EMA, which has previously been shown to be a potent regimen in GTN (Newlands et al, 2000). Three high-risk relapse patients were retreated with EMA/CO because of a long interval between treatments ( $>1$ year). None of these three have relapsed, which gives us some insight into the mechanism of chemotherapy resistance in this tumour type. Paclitaxel in combination with cisplatin and etoposide is a promising regimen in chemotherapyresistant GTN and used successfully twice in this series (Osborne et al, 2004).

The role of surgery in the management of relapse GTN is unknown. It is theoretically curative without the need for further chemotherapy, if complete excision is achieved and postoperatively the hCG falls with an appropriate half-life of 1-2 days. However, this study shows that surgery alone may be inadequate, and so the decision not to give further chemotherapy should be carefully considered. If the disease is isolated in the uterus, hysterectomy plus chemotherapy is a safe option and may reduce the duration of chemotherapy treatment (Soper, 2003). However, the majority of women maintain their fertility after chemotherapy and therefore, if a patient wishes to have children, chemotherapy alone is preferable (Bower et al, 1997).

Owing to the sensitive and specific nature of hCG in GTN, screening has been offered to relapsed patients after treatment. 
The data in this study show that most patients relapse within the first 6 months and the majority are detected by the screening programme. This finding confirms the benefit of the testing schedule for these women following chemotherapy. It is interesting that $15 \%$ patients relapsed after 2 years, which underlines the need to continue monitoring these women.

This study also investigated patients with disease refractory to high-risk therapy. These patients have poor outcome compared to those with relapsed disease and once again underlines the importance of obtaining a normal hCG with treatment for GTN.

There are two other publications in this area, both of which combined patients with resistant and relapsed disease together (Lurain and Nejad, 2005, Matsui et al, 2005). In the study by Matsui et al, only six patients with low-risk disease relapsed, and therefore, it is difficult to draw too many conclusions from this, although none have died, which is consistent with our data. The other study focused on 26 patients with high-risk disease and did not distinguish between refractory disease and relapse. As we have shown in this study, these two groups of patients have differing outcomes, making the interpretation of their results complex.

Although all patients in this series were initially diagnosed with histologically confirmed gestational trophoblastic disease, it is possible that some of the high-risk patients who were refractory or relapsed may in fact have had non-gestational hCG producing tumours that masquerade as GTN (Fisher and Newlands, 1998). In a number of isolated cases, we have performed genetic analysis to confirm the non-gestational genetic origin of the tumour. However, it is not possible to perform testing in all cases. Therefore, it is conceivable that there will be a small proportion of non-gestational tumours in this series. An additional shortcoming of this work is that none of the relapsed tumours were genetically tested to confirm that the disease was truly relapse, rather than a second unrelated tumour. However, there are a number of factors, which strongly support the theory that these patients have relapsed. These include the short period between completing chemotherapy and relapse of disease (median 4 months). Additionally, the patients with relapsed disease presented in a different manner to those at initial presentation, with a higher proportion with metastatic disease, comparatively low hCGs and low burden of disease in the uterous. Indeed no abnormality was noted in the uterous in $41 \%$ of patients at relapse. Despite this, it is not possible to completely rule out the possibility that some of these tumours were due to a second primary tumour without genetic testing.

In summary, the outcome of women with relapsed GTN is good, especially those initially treated with low-risk disease. The outcome for patients with refractory disease is less favourable. Monitoring patients after relapse is recommended for at least 5 years

\section{REFERENCES}

Bagshawe KD (1976) Risk and prognostic factors in trophoblastic neoplasia. Cancer 3: $1373-1385$

Bower M, Newlands ES, Holden L, Short D, Brock C, Rustin GJ, Begent RH, Bagshawe KD (1997) EMA/CO for high-risk gestational trophoblastic tumors: results from a cohort of 272 patients. J Clin Oncol 15: 2636-2643; Erratum in: J Clin Oncol 1997 September;15(9):3168.

Dobson LS, Lorigan PC, Coleman RE, Hancock BW (2000) Persistent gestational trophoblastic disease: results of MEA (methotrexate, etoposide and dactinomycin) as first-line chemotherapy in high risk disease and EA (etoposide and dactinomycin) as second-line therapy for low risk disease. Br J Cancer 82: $1547-1552$

Farhat F, Culine S, Theodore C, Bekradda M, Terrier-Lacombe MJ, Droz JP (1996) Cisplatin and ifosfamide with either vinblastine or etoposide as salvage therapy for refractory or relapsing germ cell tumor patients: the Institut Gustave Roussy experience. Cancer 77: $1193-1197$

Fossa SD, Waehre H, Paus E (1992) The prognostic significance of prostate specific antigen in metastatic hormone-resistant prostate cancer. $\mathrm{Br} J$ Cancer 66: $181-184$

Fisher RA, Newlands ES (1998) Gestational trophoblastic disease. Molecular and genetic studies. J Reprod Med 43: 87-97

Herzog TJ (2002) Update on the role of topotecan in the treatment of recurrent ovarian cancer. Oncologist 7: 3-10

Kohorn EI (2001) The new FIGO 2000 staging and risk factor scoring system for gestational trophoblastic disease: description and critical assessment. Int J Gynecol Cancer 11: 73-77

Lurain JR, Nejad B (2005) Secondary chemotherapy for high-risk gestational trophoblastic neoplasia. Gynecol Oncol 97: 618-623

Matsui H, Suzuka K, Yamazawa K, Tanaka N, Mitsuhashi A, Seki K, Sekiya $S$ (2005) Relapse rate of patients with low-risk gestational trophoblastic tumor initially treated with single-agent chemotherapy. Gynecol Oncol 96: $616-620$

McCaffrey JA, Mazumdar M, Bajorin DF, Bosl GJ, Vlamis V, Motzer RJ. Ifosfamide- and cisplatin-containing chemotherapy as first-line salvage therapy in germ cell tumors: response and survival. J Clin Oncol 1997; 15(7): 2559-2563

McNeish IA, Kanfer EJ, Haynes R, Giles C, Harland SJ, Driver D, Rustin GJ, Newlands ES, Seckl MJ (2004) Paclitaxel-containing high-dose chemotherapy for relapsed or refractory testicular germ cell tumours. Br J Cancer 90: 1169 - 1175

McNeish IA, Strickland S, Holden L, Rustin GJ, Foskett M, Seckl MJ, Newlands ES (2002) Low-risk persistent gestational trophoblastic disease: outcome after initial treatment with low-dose methotrexate and folinic acid from 1992 to 2000. J Clin Oncol 20: 1838-1844

Newlands ES, Begent RH, Rustin GJ, Parker D, Bagshawe KD (1983) Further advances in the management of malignant teratomas of the testis and other sites. Lancet 1: 948-951

Newlands ES, Mulholland PJ, Holden L, Seckl MJ, Rustin GJ (2000) Etoposide and cisplatin/etoposide, methotrexate, and actinomycin D (EMA) chemotherapy for patients with high-risk gestational trophoblastic tumors refractory to EMA/cyclophosphamide and vincristine chemotherapy and patients presenting with metastatic placental site trophoblastic tumors. J Clin Oncol 18: 854-859

Ngan HY (2004) The practicability of FIGO 2000 staging for gestational trophoblastic neoplasia. Int J Gynecol Cancer 14: 202-205

Osborne R, Covens A, Mirchandani D, Gerulath A (2004) Successful salvage of relapsed high-risk gestational trophoblastic neoplasia patients using a novel paclitaxel-containing doublet. J Reprod Med 49: 655-661

Papadopoulos AJ, Foskett M, Seckl MJ, McNeish I, Paradinas FJ, Rees H, Newlands ES (2002) Twenty-five years' clinical experience with placental site trophoblastic tumors. J Reprod Med 47: 460-464

Proctor SJ, Taylor PR, Angus B, Wood K, Lennard AL, Lucraft H, Carey PJ, Stark A, Iqbal A, Haynes A, Russel N, Leonard RC, Culligan D, Conn J, Jackson GH. High-dose ifosfamide in combination with etoposide and epirubicin (IVE) in the treatment of relapsed/refractory Hodgkin's disease and non-Hodgkin's lymphoma: a report on toxicity and efficacy. Eur J Haematol Suppl 2001; 64: 28-32

Rustin GJ, Newlands ES, Begent RH, Dent J, Bagshawe KD (1989) Weekly alternating etoposide, methotrexate, and actinomycin/vincristine and cyclophosphamide chemotherapy for the treatment of CNS metastases of choriocarcinoma. J Clin Oncol 7: $900-903$

Soper JT (2003) Role of surgery and radiation therapy in the management of gestational trophoblastic disease. Best Pract Res Clin Obstet Gynaecol 17: $943-957$

Thigpen JT, Vance RB, Khansur T (1993) Second-line chemotherapy for recurrent carcinoma of the ovary. Cancer 15: 71 